\title{
Review of: "Ediacaran metazoan fossils with siliceous skeletons from the Digermulen Peninsula of Arctic Norway"
}

\author{
Alan Marron
}

Potential competing interests: The author(s) declared that no potential competing interests exist.

This paper reports the discovery of three new fossil genera from the Ediacaran of Norway, preserved as three-dimensional specimens. The authors interpret these fossils as being of metazoan origin, and the results of their elemental analyses lead them to conclude that the fossils were primarily biomineralized with silica. This would make them the earliest recorded examples of metazoan biosilicification.

The authors lay out extensive evidence to support the dating of the sediments in which these fossils were found, and have produced a considerable level of detail in their analysis of the specimens themselves. I cannot comment on the technical aspects of their elemental analysis methods, but the authors do present multiple lines of support for the conclusion that the fossils were originally siliceous, both from EDS, petrographic thin-section and Laser-Raman analysis. The authors could provide comparative analyses of other fossil material from the Stahpogieddi formation, for example acritarchs or Palaeopascichnus, to confirm that the Si and O peaks in particular are confined to the three new genera, and that other elements ( $\mathrm{Al}, \mathrm{Fe}, \mathrm{Ti}$ ) are indeed derived from the host sediment. Furthermore, it would be useful to have similar analyses of confirmed biosiliceous fossils as positive controls, given that the identification of primarily biomineralized silica is so central to the paper.

The main points of discussion arise from the biological implications of the paper's conclusions that these fossils represent early biosilicifying metazoans. Assuming there is no abiotic explanation for these specimens (and I think that the authors do make a reasoned argument for them being body fossils of biological origin), then a metazoan affinity is the most likely explanation, if only based on the size of the organisms. However, to my knowledge there are no comparable examples of metazoan silica biomineralization of this type. The conical and tubular body-wall constructions are much more reminiscent of calcareous biomineralized skeletons formed by cnidarians or lophotrochozoans via an extracellular organic matrix. This is particularly the case for the growth layers and developmental sequence that the authors identify. This is in contrast to silica biomineralization being initiated intracellularly in silica deposition vesicles, and being usually comprised of elements that are added to (e.g. spicules, shell elements, mouthparts) rather than a single massive skeleton continuously growing by accretion. An alternative explanation, which is pointed towards by the authors, is that high environmental Si levels 
produced the siliceous tubes, in a manner similar to siliceous coatings of bacteria in geothermal power stations (Doi et al., Applied and Environmental Microbiology, 75(8), 2009, pp.2406-2413). In this case, the organisms would be conducting biologically-induced biomineralization, rather than biologically-controlled, which raises issues about the conclusions of the paper. How could the characteristic double-layer of a veneer and a thicker matrix form if silicification is induced and environmentally dependent? Why are there no other examples of Precambrian biota producing siliceous structures by similar means? Would these only be found in sediments from similar cold-water, high Si palaeoenvironments? Can an organism be said to be biomineralized if it only does so in specific conditions- is this not closer in theme to taphonomy than biology?

As minor comments, I would note that Neoproterozoic fossils with testate amoeba affinity are earlier examples of biomineralized eukaryotic structures (albeit not metazoan), and by comparison with extant taxa these represent good candidates for being biologically-controlled biosilicification (Porter and Knoll, Paleobiology, 26(3), 2000, pp. 360-385). The authors also claim that the resistance of the tubular and conical fossils provide strong support for them being biomineralized, however it is possible that organic structures could remain intact under such conditions. I would also take issue with their claim that biologically-induced biomineralization is characteristic of single-celled organisms: fungal hyphae or plant roots becoming coated in calcium oxalate in certain soils would be a counter-example (Raven and Knoll, Geomicrobiology Journal 27(6\&7), 2010, pp. 572-584).

Finally, the claims made in this paper should be related to recent work by Ye and colleagues (Ye et al., Earth and Planetary Science Letters 566, 2021, 116959). If silica biomineralization evolved in the Ediacaran, and was widespread to such a degree that macroscopic silica structures were being formed, then this would represent a significant biological sink for Si drawdown. This would have major implications for the timing of changes in global seawater Si levels, and the knock-on implications for biomineralization and the Cambrian explosion. 